\title{
Influência da infecção por Plasmodium vivax nos marcadores hematológicos e hepáticos em pacientes de um município da Região Amazônica brasileira
}

\section{Influence of Plasmodium vivax infection on hematological and hepatic markers in patients from a city of the Brazilian Amazon Region}

Allyson Guimarães da Costa 1,2,3,4, Andréa Monteiro Tarragô,3,5, Nadja Pinto Garcia 3,5, João Paulo Diniz Pimentel ${ }^{3}$, Walter

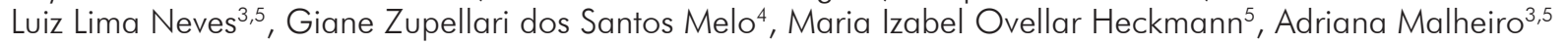

1 Universidade do Estado do Amazonas, Programa de Pós-Graduação em Medicina Tropical, Manaus, Amazonas, Brasil

2 Fundação de Medicina Tropical Dr. Heitor Vieira Dourado, Manaus, Amazonas, Brasil

${ }^{3}$ Fundação Hospitalar de Hematologia e Hemoterapia do Amazonas, Departamento de Ensino e Pesquisa, Manaus, Amazonas, Brasil

${ }^{4}$ Universidade do Estado do Amazonas, Especialização em Enfermagem em Infectologia, Manaus, Amazonas, Brasil

5 Universidade Federal do Amazonas, Instituto de Ciências Biológicas, Programa de Pós-Graduação em Imunologia Básica e Aplicada, Manaus, Amazonas, Brasil

\begin{abstract}
RESUMO
OBJETIVO: Avaliar as principais alterações hematológicas e dos marcadores hepáticos (AST e ALT) em pacientes infectados com Plasmodium vivax atendidos no Instituto de Medicina Tropical de Coari, estado do Amazonas, Brasil. MATERIAIS E MÉTODOS: Foram coletadas amostras de 77 pacientes infectados com P. vivax e de 58 indivíduos controle. $O$ hemograma completo e as dosagens de alanina aminotransferase (ALT) e aspartato aminotransferase (AST) foram realizados na Fundação Hospitalar de Hematologia e Hemoterapia do Amazonas. As análises estatísticas foram realizadas com o software GraphPad Prism v5.0. RESULTADOS: Foi observado que a maior parte dos pacientes, durante a coleta, apresentou como queixas mialgia (92\%), fraqueza (87\%), dispneia (84\%), calafrios (75\%) e febre (69\%), além de terem tido um aumento nas concentrações das enzimas hepáticas AST e ALT. Em relação às características hematológicas, observaram-se leucopenia, plaquetopenia e diminuição significativa na concentração da hemoglobina globular média e no volume globular médio. CONCLUSÃO: Os resultados sugerem que a presença dessas alterações hematológicas e o aumento na carga parasitária não influenciaram o aparecimento de casos graves da doença; porém alterações nas concentrações de AST e ALT podem indicar comprometimento hepático. Além disso, as redes de interações entre os componentes avaliados na infecção por $P$. vivax podem ser utilizadas como ferramenta de prognóstico em estudos futuros.
\end{abstract}

Palavras-chave: Malária; Plasmodium vivax; Hematologia; Marcadores Hepáticos.

\begin{abstract}
OBJECTIVE: To evaluate the main hematological changes and hepatic markers (AST and ALT) in patients infected with Plasmodium vivax treated at Instituto de Medicina Tropical de Coari, Amazonas State, Brazil. MATERIALS AND METHODS: Samples were collected from 77 patients infected with P. vivax and 58 control subjects. The complete blood count and dosages of alanine aminotransferase (ALT) and aspartate aminotransferase (AST) were performed at Fundação Hospitalar de Hematologia e Hemoterapia do Amazonas. Statistical analyzes were performed with GraphPad Prism v5.0 software. RESULTS: It was observed, during collection, that most of the patients complained of myalgia (92\%), asthenia (87\%), dyspnea (84\%), chills (75\%), and fever (69\%), besides having an increase in the concentrations of the AST and ALT enzymes. About hematological characteristics, leucopenia, thrombocytopenia and a significant decrease in mean globular hemoglobin concentration and mean globular volume were observed. CONCLUSION: The results suggest that the presence of these hematological changes and the increase in the parasite load did not influence the occurrence of severe cases of that disease; but changes in the concentrations of AST and ALT may indicate hepatic impairment. In addition, the interaction networks among the components evaluated in $P$. vivax infection can be used as a prognostic tool in future studies.
\end{abstract}

Keywords: Malaria; Plasmodium vivax; Hematology; Hepatic Markers.

\footnotetext{
Correspondência / Correspondence 


\section{INTRODUÇÃO}

A malária é causada pelo protozoário do gênero Plasmodium sp., com cinco espécies que infectam o homem: Plasmodium falciparum, Plasmodium vivax, Plasmodium malariae, Plasmodium ovale e Plasmodium knowlesi ${ }^{1,2,3}$. Possui processo patogênico diferenciado, que se inicia após a inoculação do parasito na corrente sanguínea pelo inseto vetor ${ }^{4,5}$. É considerada como um grave problema de saúde pública em mais de 100 países, sendo a doença parasitária de regiões tropicais e subtropicais que mais causa problemas sociais e econômicos no mundo $6,7,8$.

Segundo estimativas da Organização Mundial da Saúde, cerca de 3,3 bilhões de pessoas $140 \%$ da população mundial) residem em áreas de risco de transmissão da malária. Desse contingente, aproximadamente 214 milhões de pessoas foram infectadas no ano de 2015, com 438.000 mortes $^{7}$. No Brasil, cerca de 140.000 casos foram notificados, representando $41,7 \%$ dos casos nas Américas. A Região Amazônica contribui com cerca de 99,8\% das notificações, e a principal espécie causadora da malária é o P. vivax $(83,7 \% \text { dos casos })^{9,10,11}$.

○ ciclo sanguíneo ocorre de forma rápida, remodelando a estrutura das células infectadas e tornando-as mais rígidas ${ }^{12,13}$. Alterações hematológicas, principalmente na malária grave, podem ter um impacto negativo na patogênese da doença, podendo alterar o prognóstico do hospedeiro/paciente. As principais alterações observadas nos indivíduos com malária podem ser a anemia grave, os distúrbios da coagulação, a trombocitopenia proeminente e as alterações numéricas ou funcionais de leucócitos com envolvimento do baço ${ }^{14}$.

aumento das enzimas hepáticas na infecção por P. vivax é sugestivo de comprometimento hepático ${ }^{15}$. $\bigcirc$ relato de aumento, principalmente das transaminases alanina aminotransferase (ALT) e aspartato aminotransferase (AST), podem evidenciar a necrose dos hepatócitos durante o ciclo inicial da infecção no hospedeiro humano ${ }^{16,17}$.

Assim, este estudo teve por objetivo avaliar as principais alterações hematológicas e dos marcadores hepáticos (AST e ALT) em pacientes diagnosticados com malária vivax atendidos no Instituto de Medicina Tropical de Coari (IMTC), estado do Amazonas, Brasil

\section{MATERIAIS E MÉTODOS}

\section{POPULAÇÃO DO ESTUDO}

estudo foi realizado no IMTC (vinculado à Secretaria Municipal de Saúde de Coari e à Fundação de Medicina Tropical Dr. Heitor Vieira Dourado). A população de estudo compreendeu adultos, de ambos os sexos, com idade variando entre 18 e 65 anos. Os dados epidemiológicos e clínicos foram obtidos por meio de questionário estruturado, no período de fevereiro a julho de 2012.

\section{ASPECTOS ÉTICOS}

Este estudo foi aprovado pelo Comitê de Ética em Pesquisa da Fundação Hospitalar de Hematologia e Hemoterapia do Amazonas (Hemoam) em 31 de outubro de 2011, com o protocolo CAEE $n^{\circ}$ 0014.0.112.000-11, estando de acordo com a Resolução n 466/12 do Conselho Nacional de Saúde para pesquisas envolvendo seres humanos.

\section{AMOSTRAGEM}

A amostra foi composta por 77 pacientes com quadro de malária aguda não grave, causada por P. vivax, atendidos no IMTC; e 58 indivíduos enquadrados no grupo controle (GC), escolhidos ao acaso, com pesquisa de plasmódios negativa, com idade entre 18 e 65 anos. Os pacientes e indivíduos do GC foram negativos para os vírus da hepatite B, da hepatite $\mathrm{C}$ e HIV por testes sorológicos (imunoensaio quimioluminescente automatizado - ARCHITECT i2000SR Plus, Abbott Diagnostics), no Laboratório de Sorologia da Hemoam.

\section{DIAGNÓSTICO E CONTAGEM DA PARASITEMIA}

diagnóstico da malária dos pacientes infectados com $P$. vivax foi realizado pelo exame da gota espessa de sangue, como recomendado pelo Ministério da Saúde ${ }^{18}$. A monoinfecção por P. vivax foi confirmada por reação em cadeia da polimerase via transcriptase reversa $(R T-P C R)^{19}$. A parasitemia foi estimada pela análise semiquantitativa, com os resultados expressos em: +/2 (40-60/100 parasitos por campo); + (1 parasito/campo); ++ (2-20 parasitos/campo); +++ (21-200 parasitos/campo); e $++++(+200$ parasitas/ campo). Com os dados em cruzes, a parasitemia em milímetro cúbico pode ser estimada conforme descrito pelo Ministério da Saúde ${ }^{18}$ : +/2 = 200-300 parasitos, $+\quad=301-500$ parasitos; $++=501-10.000$ parasitos; $+++=10.001-100.000$ parasitos; ++++ $=100.000$ ou mais parasitos.

\section{AVALIAÇÃO CLÍNICA DOS PACIENTES}

Os pacientes incluídos foram avaliados, após o diagnóstico por enfermeiro e/ou médico do IMTC. Os resultados obtidos na anamnese e no exame físico foram inseridos no questionário próprio do estudo.

\section{TESTES HEMATOLÓGICOS}

Para a avaliação hematológica, foi realizada a contagem global dos leucócitos (GB), células vermelhas $(G V)$, das plaquetas (PLAQ), determinação do hematócrito $(H T)$, dosagem de hemoglobina $(H B)$ e índices hematimétricos em contador hematológico $A B X$ Micros 60 (HORIBA).

\section{TESTES BIOQUÍMICOS - TRANSAMINASES (AST E ALT)}

As dosagens das concentrações das transaminases (AST e ALT) foram realizadas com os kits Aspartate Aminotransferase AEROSET c8000 (Abbott Laboratories) e Alanine Aminotransferase AEROSET c8000 (Abbott Laboratories), com método automatizado utilizando equipamento ARCHITECT c8000 System Clinical Biochemistry Analyzer (Abbott Diagnostics). 


\section{ANÁLISES ESTATÍSTICAS E REDES DE INTERAÇÕES}

As análises estatísticas dos dados foram realizadas com o software GraphPad Prism v5.0 (San Diego, Califórnia, EUA). Inicialmente foram realizados testes para verificar a normalidade dos dados por intermédio do teste de Shapiro-Wilk. Todos os dados apresentaram uma distribuição não paramétrica, e, portanto, as análises comparativas dos valores entre dois ou três grupos de dados foram realizadas com o teste de Mann-Whitney ou Kruskal-Wallis, respectivamente. A significância estatística foi definida em ambos os casos em $p<0,05$.

A elaboração das redes (networks) e demonstração das interações complexas entre os parâmetros avaliados foi realizada a partir da associação desses marcadores em cada grupo clínico. Foi realizado o teste de correlação de Spearman para avaliar a correlação significativa entre o valor absoluto e as concentrações dos componentes, com o auxílio do software estatístico. Com os dados gerados após a análise de correlação, foi realizada a construção das redes com o software Cytoscape v3.3.1 (Cytoscape Consortium San Diego, Califórnia, EUA). A forma e a espessura das linhas foram ajustadas para representar os tipos de correlações existentes (positivas ou negativas), além da intensidade dessas correlações.

\section{RESULTADOS}

\section{CARACTERÍSTICAS EPIDEMIOLÓGICAS, PARASITÁRIAS E CLIINICAS DOS PACIENTES DIAGNOSTICADOS COM MALÁRIA VIVAX}

A média de idade foi maior no grupo de pacientes $(37,06 \pm 14,32)$ em relação ao $\mathrm{GC}(29,41 \pm 8,45)$ ( $p=0,003)$. Quanto ao gênero, foi observado predomínio do sexo masculino (69\%) nos pacientes com malária, sendo notado o inverso no $G C$, onde foi observada maior predominância do sexo feminino (67\%) ( $<<0,0001$ ). Observou-se que a maioria (51\%) dos indivíduos relatou ter tido entre duas e cinco reinfecções (Tabela 1). A parasitemia de $+/ 2$ e ++ foi observada em mais de dois terços dos pacientes (74\%) com sintomatologia básica da malária. Mialgia foi a queixa mais frequente entre os pacientes (92\%), seguindo-se de astenia (97\%), cefaleia (84\%), calafrios $(75 \%)$ e febre $(69 \%)$ no momento da coleta (Tabela 2).

Tabela 1 - Dados epidemiológicos dos pacientes com malária vivax e grupo controle atendidos no IMTC, estado do Amazonas, Brasil, no período de fevereiro a julho de 2012

\begin{tabular}{lcc}
\hline Características demográficas e epidemiológicas & Grupo controle $(\mathrm{N}=58)$ & Pacientes com P. vivax (N=77) \\
\hline Masculino/Feminino & $19 / 39$ & $53 / 24$ \\
Idade (média $\pm \mathrm{DP})$ & $29,41 \pm 8,45$ & $37,06 \pm 14,32$ \\
Número de episódios prévios de malária & - & $5,57 \pm 6,77(1-40)$ \\
Número de infecções & - & $15(19 \%)$ \\
$\quad$ Primoinfectado & - & $39(51 \%)$ \\
$<5$ infecções & - & $23(30 \%)$ \\
$>5$ infecções & & \\
\hline
\end{tabular}

DP: Desvio padrão.

Tabela 2 - Características parasitológicas e parâmetros clínicos dos pacientes com malária vivax e grupo controle atendidos no IMTC, estado do Amazonas, Brasil, no período de fevereiro a julho de 2012

\begin{tabular}{lccc}
\hline & Características parasitológicas & \multicolumn{2}{c}{ Pacientes com P. vivax (N=77) } \\
\cline { 2 - 4 } Parasitemia & Parasitas $/ \mathrm{mm}^{3}$ & $N$ & $\%$ \\
\hline $200-300$ & $+/ 2$ & 20 & 26 \\
$301-500$ & + & 18 & 23 \\
$501-10.000$ & ++ & 37 & 48 \\
$10.000-100.000$ & +++ & 2 & 3 \\
\hline & & Parâmetros clínicos \\
& & $N$ & $\%$ \\
\hline Febre durante a coleta sanguínea & 52 & 69 \\
Mialgia nos últimos três dias & & 71 & 92 \\
Dispneia nos últimos três dias & 64 & 84 \\
Calafrio nos últimos três dias & 58 & 75 \\
Fraqueza nos últimos três dias & 67 & 87 \\
\hline
\end{tabular}


ALTERAÇÕES HEMATOLÓGICAS E HEPÁTICAS (AST E ALT) EM PACIENTES INFECTADOS POR Plasmodium vivax

Como sumarizado na tabela 3, nos pacientes com malária vivax, observou-se diminuição significativa da GB, da concentração de hemoglobina corpuscular média (MCHC), do volume corpuscular médio (VCM), de PLAQ e da variação no tamanho das plaquetas (PDW), além do aumento significativo do volume plaquetário médio (VPM). Ademais, a análise dos marcadores hepáticos demonstrou aumento significativo da transaminase AST ( $p=0,0093)$, sendo significativamente maior no grupo de pacientes com malária vivax quando comparado ao GC. Não foi observada significância estatística nos níveis de ALT entre os grupos estudados.

ASPECTOS HEMATOLÓGICOS E HEPÁTICOS (AST E ALT) NOS SUBGRUPOS CLÍNICOS DE PACIENTES COM MALÁRIA VIVAX

As alterações hematológicas e dos marcadores hepáticos (AST e ALT) foram analisadas ainda nos subgrupos de pacientes com malária vivax. Inicialmente, os pacientes foram separados em grupos, de acordo com a parasitemia (baixa $[+/ 2$ e +$]$ e alta $[++$ e $+++]$ ) e o número de infecções (primoinfectado, $\leq 5$ infecções e $>5$ infecções). Em relação à parasitemia, observou-se diminuição significativa de HT, HB e GV nos pacientes com alta carga parasitária. Verificou-se um aumento significativo $(p=0,0197)$ na variação do PDW dos pacientes primoinfectados em relação aos indivíduos com mais de uma infecção (Tabela 4).

\section{INTERAÇÕES ENTRE OS MARCADORES HEMATOLÓGICOS E HEPÁTICOS (AST E ALT) NA MALÁRIA VIVAX}

Os indivíduos não infectados apresentaram uma rede com várias interações entre os marcadores hematológicos e hepáticos (AST e ALT) avaliados. O hematócrito, a hemoglobina e os glóbulos vermelhos estavam fortemente associados, enquanto que houve correlação forte entre o VCM e a MCHC, além do ALT e do AST. Os demais marcadores se correlacionaram de forma moderada e fraca, com a presença de correlações negativas entre PLAQ, VPM, PDW, GB, VCM e variação no tamanho das hemácias (RDW) no GC (Figura 1A). Quando observados os dados de correlação dos pacientes com malária vivax, notou-se a perda de correlações moderadas entre os marcadores e a ausência de interação do PDW com os demais componentes analisados. Além disso, o grupo infectado com P. vivax apresentou aumento nas interações $G B$, GV, HB, VCM e PLAQ (Figura 1B).

Tabela 3 - Características hematológicas e hepáticas dos pacientes com malária vivax e grupo controle atendidos no IMTC, estado do Amazonas, Brasil, no período de fevereiro a julho de 2012

\begin{tabular}{|c|c|c|c|}
\hline Características & $\begin{array}{l}\text { Grupo controle } \\
\qquad(N=58)\end{array}$ & $\begin{array}{l}\text { Pacientes com P. vivax } \\
\qquad(\mathrm{N}=77)\end{array}$ & $p^{*}$ \\
\hline \multicolumn{4}{|l|}{ Hematológicas (média \pm DP) } \\
\hline HT (\%) & $38,81 \pm 4,93$ & $38,43 \pm 5,54$ & 0,8224 \\
\hline HB, níveis $\mathrm{g} / \mathrm{dL}$ & $13,13 \pm 1,66$ & $13,11 \pm 1,87$ & 0,9982 \\
\hline GB (unid. $\times 10^{3} / \mathrm{mm}^{3}$ ) & $5,97 \pm 1,66$ & $4,54 \pm 1,47$ & $<0,0001$ \\
\hline GV (unid. x 106/mm ${ }^{3}$ ) & $4,12 \pm 0,64$ & $4,15 \pm 0,60$ & 0,5754 \\
\hline VCM (fL) & $94,47 \pm 7,16$ & $89,09 \pm 19,24$ & 0,0272 \\
\hline HCM (pg) & $31,96 \pm 2,76$ & $31,78 \pm 2,96$ & 0,3571 \\
\hline $\mathrm{CHCM}(\mathrm{g} / \mathrm{dL})$ & $33,73 \pm 1,13$ & $34,19 \pm 1,16$ & 0,0373 \\
\hline RDW (\%) & $13,99 \pm 1,92$ & $13,66 \pm 2,40$ & 0,9486 \\
\hline PLAQ (unid. x 106/mm³) & $237,30 \pm 84,04$ & $118,30 \pm 58,71$ & $<0,0001$ \\
\hline VPM (fL) & $7,37 \pm 2,24$ & $8,44 \pm 2,42$ & 0,0007 \\
\hline PDW (\%) & $14,30 \pm 2,31$ & $13,41 \pm 2,32$ & 0,0111 \\
\hline \multicolumn{4}{|l|}{ Hepáticas (média \pm DP) } \\
\hline AST (U/L) & $20,14 \pm 8,84$ & $55,27 \pm 252,40$ & 0,0093 \\
\hline ALT $(U / L)$ & $20,66 \pm 15,18$ & $42,87 \pm 167,50$ & 0,4493 \\
\hline
\end{tabular}




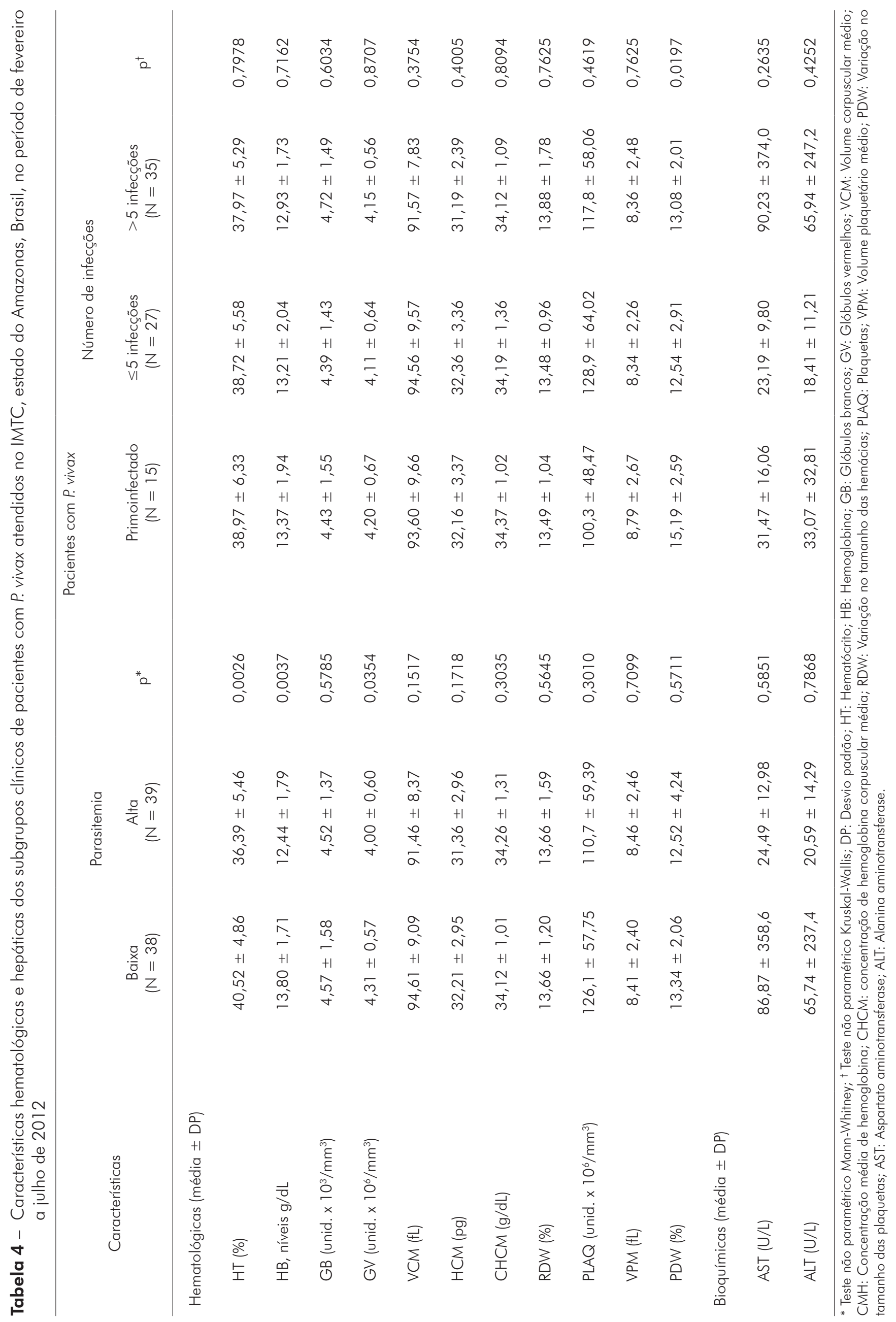


A

Grupo controle

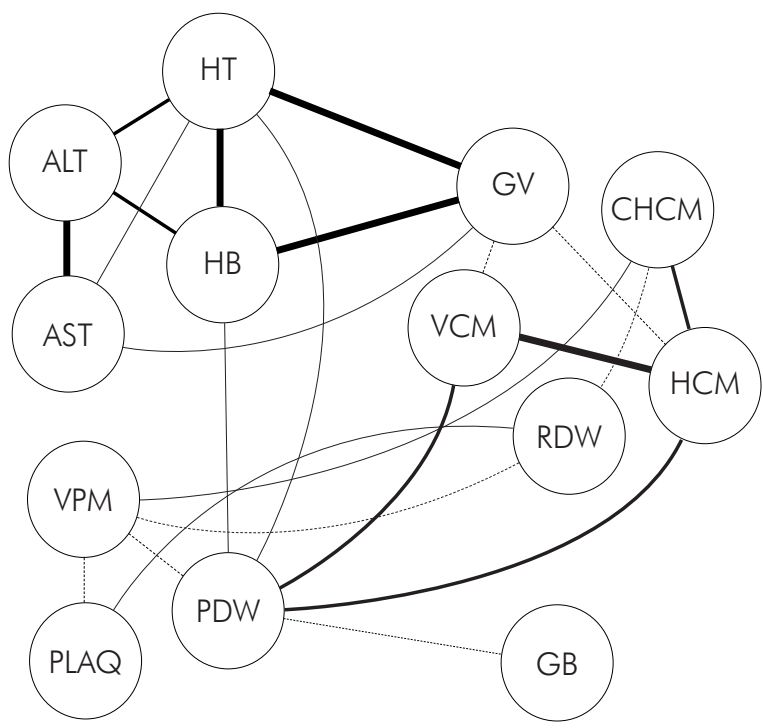

Correlações positivas

Correlação fraca $(r=\leq 0,35)$

Correlação moderada $(r=0,36-0,67)$

Correlação forte $(r=\geq 0,68)$
B

Pacientes com P. vivax

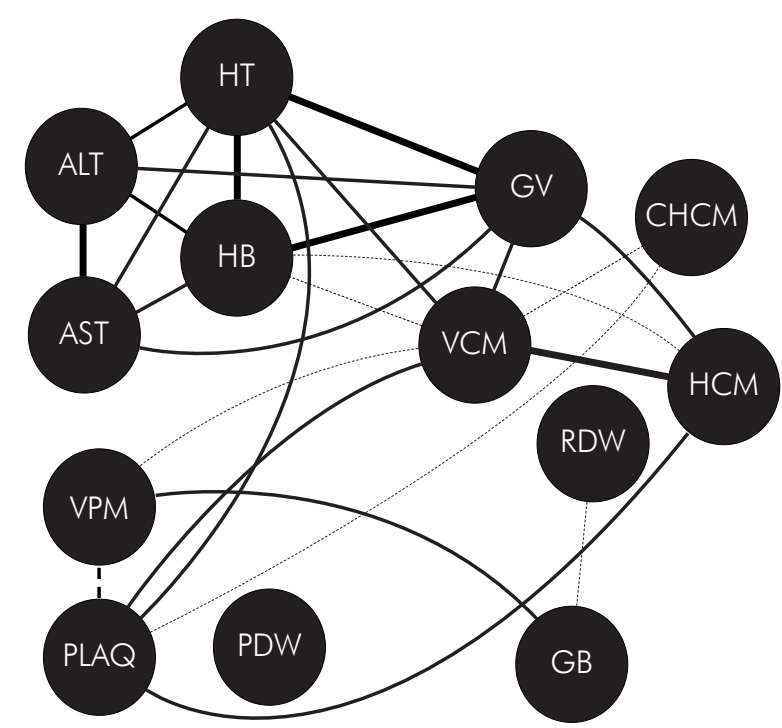

Correlações negativas

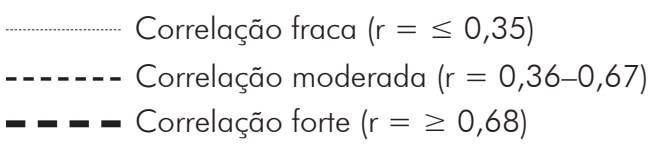

Os componentes hematológicos (HT: Hematócrito; HB: Hemoglobina; GB: Glóbulos brancos; GV: Glóbulos vermelhos; VCM: Volume corpuscular médio; $\mathrm{CMH}$ : Concentração média de hemoglobina; CHCM: Concentração de hemoglobina corpuscular média; RDW: Variação no tamanho das hemácias; PLAQ: Plaquetas; VPM: Volume plaquetário médio; PDW: Variação no tamanho das plaquetas) e hepáticos (AST: Aspartato aminotransferase; ALT: Alanine aminotransferase) foram analisados em sangue total e soro de pacientes infectados com $P$. vivax e indivíduos controles sem a doença. Cada linha de ligação representa uma correlação significativa entre um par de marcadores. As linhas contínuas representam correlações positivas, enquanto as linhas tracejadas representam correlações negativas ( $p<0,05)$. $\bigcirc$ grau de significância é representado pela espessura da linha. As correlações foram obtidas por intermédio do teste de Spearman; o valor do " $r$ " e "p" foram utilizados para classificar as conexões como fraca $(r \leq 0,35, p<0,05)$, moderada $(r=0,36-0,67, p=0,001)$ ou forte $(r \geq 0,68, p<0,0001)$. A ausência da linha indica a não existência de correlação.

Figura 1 - Redes de interações entre os marcadores hematológicos e hepáticos no grupo controle e pacientes com P. vivax atendidos no IMTC, estado do Amazonas, Brasil, no período de fevereiro a julho de 2012

\section{DISCUSSÃO}

A malária é uma doença febril aguda, com processo patogênico diferenciado e relacionado aos fatores biológicos do parasito e do hospedeiro. Neste trabalho, foram descritas as alterações clínicas, hematológicas e hepáticas (AST e ALT) causadas pela infecção por $P$. vivax em pacientes diagnosticados com malária e que não haviam iniciado o tratamento recomendado pelo Ministério da Saúde.

A sintomatologia básica da malária foi observada nos pacientes estudados, sendo as queixas mais frequentes mialgia, fraqueza, cefaleia, calafrio e febre. Para Costa et al. ${ }^{20}$, os sinais e sintomas da malária são constituídos, principalmente, por uma tríade clássica composta por febre-calafrio-cefaleia. No entanto, Rosas ${ }^{21}$ alertou para a familiaridade desses sintomas com outras doenças febris, como dengue, febre de Chikungunya e febre por Zika vírus. No estudo de Franklin et al. ${ }^{22}$, realizado na cidade de Manaus, estado do Amazonas, foi observado que os pacientes infectados com P. vivax foram acometidos principalmente por febre, mialgia, calafrio e cefaleia, o que corrobora com os achados do presente estudo, com exceção de febre e cefaleia, que foram pouco relatadas. Apesar de os pacientes incluídos nesse estudo não apresentarem sintomatologia da malária grave, estudos recentes têm associado a infecção por P. vivax com casos graves e complicações na malária $^{9,23,24}$.

Quadros anêmicos são comuns em pacientes com malária grave ocasionada por P. vivax. Em geral, os valores de HT, HB e GV estão diminuídos nesses indivíduos $17,23,25$. Em alguns casos, os pacientes são submetidos à transfusão de concentrados de hemácias devido à gravidade da anemia ${ }^{26}$. Embora a literatura indique os processos anêmicos na malária, neste estudo, o GV, ○ HT, a $\mathrm{HB}$ e os índices hematimétricos apresentaram valores similares entre os grupos estudados, com exceção do VCM, que foi menor no grupo de pacientes com malária vivax. O quantitativo similar desses componentes entre os grupos pode sugerir a manutenção das interações estudadas. Os valores de HT, HB e do número de eritrócitos foram menores apenas nos indivíduos com alta carga parasitária, o que já é conhecido e descrito na literatura ${ }^{17,23}$. Sabe-se que $\circ$ aumento da carga parasitária pode acarretar diminuição no número de GV, devido à ruptura dessas células durante os ciclos assexuados, ao sequestro de hemácias infectadas pelo baço e à formação de $\operatorname{rosetas}^{27,28,29}$. 
Os valores de GB foram menores no grupo de pacientes com malária. Dados da literatura descreveram a redução no quantitativo de $G B$ em pacientes com malária ${ }^{30,31}$. No entanto, em indivíduos com malária grave infectados com $P$. vivax, foi observado aumento no número de leucócitos ${ }^{26}$. No estudo de Tangpukdee et al. ${ }^{32}$, foi exposto ainda que GB foi menor que GV em indivíduos com P. vivax, com aproximação dos valores normais observados no grupo não infectado, após o tratamento, o que vem de encontro aos dados observados neste estudo. A diminuição dos GB é observada no início da infecção, com leucocitose transitória durante os paroxismos febris da malária, estando relacionada à carga parasitária $^{33,34}$.

A trombocitopenia foi descrita nas amostras de pacientes com malária no presente estudo, com diminuição do VPM e aumento da PDW, inclusive no grupo de pacientes primoinfectados. A plaquetopenia é comum na patogênese da malária, principalmente na infecção por P. vivax ${ }^{17,35}$. Pacientes infectados com $P$. vivax, em geral, apresentam diminuição no número de PLAQ, devido à ativação da cascata de coagulação, além dos processos inflamatórios inerentes à doença ${ }^{24,36}$. Esse decréscimo no quantitativo de PLAQ e na PDW, associado ao aumento do VPM, podem explicar o aumento das correlações na análise de rede entre os componentes associados às PLAQ, com perda de interação da PDW com os demais marcadores hematológicos e hepáticos estudados.

Os dados deste estudo sugerem ainda comprometimento hepático nesses pacientes, com aumento das enzimas hepáticas AST e ALT. Dados similares foram observados por Onyesom e Onyemakonor ${ }^{15}$ e Elbadawi et al. ${ }^{37}$, que descreveram - aumento das enzimas em pacientes da Nigéria e em mulheres grávidas do Sudão infectados com P. falciparum. $O$ relato de aumento das transaminases
AST e ALT foi observado em pacientes com P. vivax, evidenciando a necrose dos hepatócitos durante a infecção ${ }^{16,17}$. Estudos prévios realizados com pacientes com malária grave na Fundação de Medicina Tropical do Amazonas e do Hospital São Gabriel, no estado de Rondônia, descreveram aumento de AST e ALT, corroborando com os resultados do presente estudo $23,26,38$. Vale ressaltar que, apesar do aumento nas transaminases AST e ALT no grupo de pacientes com malária vivax, não foi observado aumento nas interações desses marcadores com os componentes hematológicos, o que exclui, dessa forma, esses componentes como potenciais biomarcadores da infecção e do agravamento da doença.

\section{CONCLUSÃO}

Os resultados demonstraram que formas diferentes de abordagem dos marcadores hematológicos e hepáticos podem ser úteis para a avaliação dos pacientes. Além disso, reforçaram que o ganho e/ou perda de interação entre os componentes avaliados na infecção por $P$. vivax podem ser utilizados como ferramenta de prognóstico em estudos futuros; enquanto a diminuição nos valores de $H T, H B$ e GB nos indivíduos com alta parasitemia evidenciaram a utilização desses marcadores como instrumento para o acompanhamento da evolução do quadro clínico e de formas graves da malária vivax.

\section{AGRADECIMENTOS}

Ao IMTC e à Hemoam, pelo suporte no recrutamento dos pacientes e colaboração na realização dos exames laboratoriais.

\section{APOIO FINANCEIRO}

Fundação de Amparo à Pesquisa do Estado do Amazonas (Fapeam) e Conselho Nacional de Desenvolvimento Científico e Tecnológico (CNPq).

\section{REFERÊNCIAS}

1 Ministério da Saúde (BR). Secretaria de Vigilância em Saúde. Departamento de Vigilância Epidemiológica. Ações de controle da Malária: manual para profissionais de Saúde na Atenção Básica. Brasília: Ministério da Saúde; 2006. 52 p. (Série A. Normas e manuais técnicos).

2 Cox-Singh J, Davis TME, Lee K-S, Shamsul SSG, Matusop A, Ratnam $S$, et al. Plasmodium knowlesi malaria in humans is widely distributed and potentially life threatening. Clin Infect Dis. 2008 Jan;46(2): 165-71.

3 Crawley J, Chu C, Mtove G, Nosten F. Malaria in children. Lancet. 2010 Apr;375(9724):1468-81.

4 Miller LH, Baruch DI, Marsh K, Doumbo OK. The pathogenic basis of malaria. Nature. 2002 Feb;415(6872):673-9.
5 Schofield L, Grau GE. Immunological processes in malaria pathogenesis. Nat Rev Immunol. 2005 Sep;5(9):722-35.

6 Greenwood BM, Fidock DA, Kyle DE, Kappe SHI, Alonso PL, Collins FH, et al. Malaria: progress, perils, and prospects for eradication. J Clin Invest. 2008 Apr;1 18(4): 1266-76.

7 World Health Organization. World Malaria Report. Geneva: WHO; 2015.

8 Parise EV. Malária grave em Palmas, Estado do Tocantins: relato de caso. Rev Soc Bras Med Trop. 2009 jul-ago;42(4):463-8.

9 Oliveira-Ferreira J, Lacerda MVG, Brasil P, Ladislau JLB, Tauil PL, Daniel-Ribeiro CT. Malaria in Brazil: an overview. Malar J. 2010 Apr; 9:115. 
10 Ministério da Saúde (BR). Secretaria de Vigilância em Saúde. Situação epidemiológica da malária no Brasil, 2012 e 2013. Bol Epidemiol. 2015;46(43): $1-5$.

11 Sampaio VS, Siqueira AM, Alecrim MGC, Mourão MPG, Marchesini PB, Albuquerque BC, et al. Malaria in the State of Amazonas: a typical Brazilian tropical disease influenced by waves of economic development. Rev Soc Bras Med Trop. 2015 Jun;48 Suppl 1:4-11.

12 Cowman AF, Berry D, Baum J. The cellular and molecular basis for malaria parasite invasion of the human red blood cell. J Cell Biol. 2012 Sep; 198(6):961-71.

13 Prudêncio M, Mota MM, Mendes AM. A toolbox to study liver stage malaria. Trends Parasitol. 2011 Dec;27(12):565-74.

14 Akinosoglou KS, Solomou EE, Gogos CA. Malaria: a haematological disease. Hematology. 2012 Mar;17(2):106-14.

15 Onyesom I, Onyemakonor N. Levels of parasitaemia and changes in some liver enzymes among malarial infected patients in Edo-Delta Region of Nigeria. Curr Res J Biol Sci. 2011 Mar;3(2):78-81.

16 Kochar DK, Singh P, Agarwal P, Kochar SK, Pokharna R, Sareen PK. Malarial hepatitis. J Assoc Physicians India. 2003 Nov;51:1069-72.

17 Lacerda MVG, Mourão MPG, Alexandre MAA, Siqueira AM, Magalhães BML, Martinez-Espinosa $F E$, et al. Understanding the clinical spectrum of complicated Plasmodium vivax malaria: a systematic review on the contributions of the Brazilian literature. Malar J. 2012 Jan; 1 1:12.

18 Ministério da Saúde (BR). Secretaria de Vigilância Epidemiológica. Manual de diagnóstico laboratorial da malária. Brasília: Ministério da Saúde; 2005.

19 Perandin F, Manca N, Calderaro A, Piccolo G, Galati L, Ricci L, et al. Development of a real-time PCR assay for detection of Plasmodium falciparum, Plasmodium vivax, and Plasmodium ovale for routine clinical diagnosis. J Clin Microbiol. 2004 Mar;42(3):1214-9.

20 Costa AP, Bressan CS, Pedro RS, Valls-de-Souza $R$, Silva S, Souza PR, et al. Diagnóstico tardio de malária em área endêmica de dengue na extraAmazônia Brasileira: experiência recente de uma unidade sentinela no estado do Rio de Janeiro. Rev Soc Bras Med Trop. 2010 set-out;43(5):571-4.

21 Rosas MJ. Prevenção da malária. Rev Port Clin Geral. 2003;19(3):263-6.

22 Franklin BS, Vitorino BLF, Coelho HC, MenezesNeto A, Santos MLS, Campos FMF, et al. Plasma circulating nucleic acids levels increase according to the morbidity of Plasmodium vivax malaria. PLoS One. 2011 Jan;6(5):e19842.
23 Andrade BB, Reis-Filho A, Souza-Neto SM, Clarêncio J, Camargo LMA, Barral A, et al. Severe Plasmodium vivax malaria exhibits marked inflammatory imbalance. Malar J. 2010 Jan;9:13.

24 Lacerda MVG, Mourão MPG, Coelho HCC, Santos JB. Thrombocytopenia in malaria: who cares? Mem Inst Oswaldo Cruz. 2011 Aug;106 Suppl 1:52-63.

25 Fernandes AAM, Carvalho LJM, Zanini GM, Ventura AMRS, Souza JM, Cotias PM, et al. Similar cytokine responses and degrees of anemia in patients with Plasmodium falciparum and Plasmodium vivax infections in the Brazilian Amazon region. Clin Vaccine Immunol. 2008 Apr; 15(4):650-8.

26 Alexandre MA, Ferreira CO, Siqueira $A M$, Magalhães BL, Mourão MPG, Lacerda MV, et al. Severe Plasmodium vivax malaria, Brazilian Amazon. Emerg Infect Dis. 2010 Oct;16(10): $1611-4$.

27 Miller LH, Ackerman HC, Su X, Wellems TE. Malaria biology and disease pathogenesis: insights for new treatments. Nat Med. 2013 Feb;19(2): 156-67.

28 Lacerda MVG, Fragoso SCP, Alecrim MGC, Alexandre MAA, Magalhães BML, Siqueira AM, et al. Postmortem characterization of patients with clinical diagnosis of Plasmodium vivax malaria: to what extent does this parasite kill? Clin Infect Dis. 2012 Oct;55(8):e67-74.

29 Carvalho BO, Lopes SCP, Nogueira PA, Orlandi PP, Bargieri DY, Blanco YC, et al. On the cytoadhesion of Plasmodium vivax-infected erythrocytes. J Infect Dis. 2010 Aug;202(4): 638-47.

30 Rodríguez-Morales AJ, Sánchez E, Arria M, Vargas M, Piccolo C, Colina R, et al. White blood cell counts in Plasmodium vivax malaria. J Infect Dis. 2005 Nov; 192(9): 1675-6.

31 Tchinda GG, Atashili J, Achidi EA, Kamga HL, Njunda $A L$, Ndumbe PM. Impact of malaria on hematological parameters in people living with HIV/ AIDS attending the Laquintinie Hospital in Douala, Cameroon. PLoS One. 2012 Jul;7(7):e40553.

32 Tangpukdee N, Yew H-S, Krudsood S, Punyapradit $\mathrm{N}$, Somwong W, Looareesuwan $\mathrm{S}$, et al. Dynamic changes in white blood cell counts in uncomplicated Plasmodium falciparum and P. vivax malaria. Parasitol Int. 2008 Dec;57(4):490-4.

33 McKenzie FE, Prudhomme WA, Magill AJ, Forney JR, Permpanich B, Lucas C, et al. White blood cell counts and malaria. J Infect Dis. 2005 Jul;192(2): 323-30.

34 Kotepui M, Phunphuech B, Phiwklam N, Chupeerach C, Duangmano S. Effect of malarial infection on haematological parameters in population near Thailand-Myanmar border. Malar J. 2014 Jun; 13:218. 
35 Kochar DK, Das A, Kochar A, Middha S, Acharya J, Tanwar GS, et al. Thrombocytopenia in Plasmodium falciparum, Plasmodium vivax and mixed infection malaria: a study from Bikaner (Northwestern India). Platelets. 2010 Nov;21(8): 623-7.

36 Francischetti IMB, Seydel KB, Monteiro RQ. Blood coagulation, inflammation, and malaria. Microcirculation. 2008 Feb; 15(2):81-107.
37 Elbadawi NEE, Mohamed MI, Elzaki H, Ounsa MAAGE, Mohamed EY, Ibrahim EK. The effect of malaria on biochemical liver function parameters in Sudanese pregnant women. J Physiobiochem Metab. 2012 Sep; 1:2.

38 Mendonça VRR, Queiroz ATL, Lopes FM, Andrade BB, Barral-Netto M. Networking the host immune response in Plasmodium vivax malaria. Malar J. 2013 Jan; 12:69. 